\title{
Structural plasticity in the bilingual brain
}

\section{Proficiency in a second language and age at acquisition affect grey-matter density.}

umans have a unique ability to learn more than one language - a skill that is thought to be mediated by functional (rather than structural) plastic changes in the brain ${ }^{1}$. Here we show that learning a second language increases the density of grey matter in the left inferior parietal cortex and that the degree of structural reorganization in this region is modulated by the proficiency attained and the age at acquisition. This relation between grey-matter density and performance may represent a general principle of brain organization.

We used a whole-brain unbiased objective technique, known as voxel-based morphometry $(\mathrm{VBM})^{2,3}$, to investigate structural plasticity in healthy right-handed English and Italian bilinguals.

To test for differences in the density of grey and white matter between bilinguals and monolinguals, we recruited 25 monolinguals who had had little or no exposure to a second language; 25 'early' bilinguals, who had learned a second European language before the age of 5 years and who had practised it regularly since; and 33 'late' bilinguals, who had learned a second European language between the ages of 10 and 15 years and practised it regularly for at least 5 years. All volunteers for this test were native English speakers of comparable age and level of education.

Voxel-based morphometry revealed that grey-matter density in the inferior parietal cortex was greater in bilinguals than monolinguals (Fig. 1a). This effect was significant in the left hemisphere $(x=-45, y=-59$, $z=48 ; Z$-score $=7.1 ; P<0.05$, corrected for multiple comparisons across the whole brain) and a trend was also evident in the right hemisphere $(x=56, y=-53, z=42 ; Z$-score $=3.4$; $P<0.001$, uncorrected). Although increased grey-matter density in the inferior parietal cortex was common to both early and late bilinguals, the effect was greater in the early bilinguals in the left $(x=-48, y=-62$, $z=44 ; Z$-score $=3.5 ; P<0.001$, uncorrected) and right $(x=45, \quad y=-65, \quad z=47 ; \quad Z$ score $=3.5 ; \quad P<0.001$, uncorrected) hemispheres. No other significant effects were detected in either grey or white matter.

We next investigated whether there was a relation between brain structure and proficiency in the second language and age at acquisition. We tested 22 native Italian speakers who had learned English as a second language at an age between 2 and 34 years. Second-language reading, writing, speech comprehension and production were assessed using a battery of standardized neuropsychological tests (see supplementary information). We found that overall proficiency, as
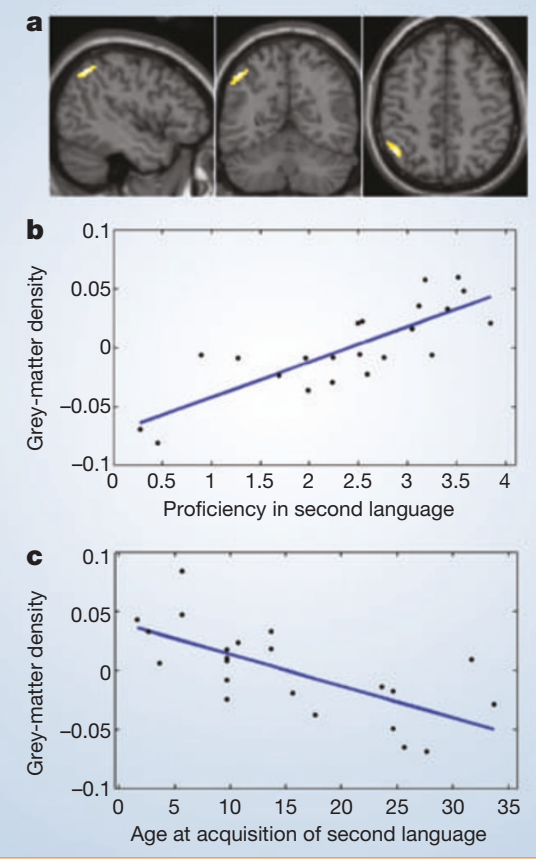

Figure 1 Structural reorganization in the bilingual brain. a, Sagittal $(x=-45)$, coronal $(y=-59)$ and axial $(z=48)$ view of the left inferior parietal region, which has increased grey-matter density in bilinguals relative to monolinguals. $\mathbf{b}$, Grey-matter density, measured as cubic millimetres of grey matter per voxel in the left inferior parietal region, as a function of second-language proficiency. Second-language proficiency was estimated for each subject from a battery of standardized neuropsychological tests, using principal component analysis (for details, see supplementary information). c, Grey-matter density, measured as for $\mathbf{b}$, as a function of age at acquisition.

indexed by principal component analysis, correlated negatively with age at acquisition $(P<0.01 ; r=-0.855)$. Remarkably, VBM revealed that second-language proficiency correlated with grey-matter density in exactly the same left inferior parietal region that we had already identified $(x=-48, y=-59$, $z=46 ; Z$-score $=4.1 ; P<0.05$, corrected after $10-\mathrm{mm}$ small-volume correction; Fig. 1b). In addition, grey-matter density in this region correlated negatively with the age of acquisition of the second language $(x=-50$, $y=-58, \quad z=42 ; \quad Z$-score $=3.2 ; \quad P<0.05$, corrected after $10-\mathrm{mm}$ small-volume correction; Fig. 1c). There were no other significant effects in grey or white matter.

We have therefore identified an increase in the density of grey matter in the left inferior parietal cortex of bilinguals relative to monolinguals, which is more pronounced in early rather than late bilinguals, and have also shown that the density in this region increases with second-language proficiency but decreases as the age of acquisition increases.

These effects could result from a genetic predisposition to increased density, or from a structural reorganization induced by experience ${ }^{4}$. Early bilinguals probably acquire a second language through social experience, rather than as a result of a genetic predisposition. Our findings therefore suggest that the structure of the human brain is altered by the experience of acquiring a second language.

The inferior parietal region that is associated with second-language acquisition corresponds exactly to an area that has been shown by functional imaging to become activated during verbal-fluency tasks ${ }^{5,6}$. Whether grey-matter reorganization in this region is related to changes in neuropil, neuronal size, dendritic or axonal arborization will be revealed by methods other than whole-brain magnetic resonance imaging.

These results are consistent with growing evidence that the human brain changes structurally in response to environmental demands - for example, structure is already known to alter as a function of learning in domains other than language ${ }^{7,8}$. We have shown that the degree of this structural reorganization in bilinguals is correlated with their secondlanguage performance. The relationship between grey-matter density and performance discovered here could be an example of a more general structure-function principle that extends beyond the domain of language.

Andrea Mechelli*, Jenny T. Crinion*,

Uta Noppeney ${ }^{\star}$, John O’Doherty ${ }^{\star}$,

John Ashburner ${ }^{\star}$, Richard S. Frackowiak ${ }^{\star} \dagger$, Cathy J. Price ${ }^{*}$

${ }^{\star}$ Wellcome Department of Imaging Neuroscience,

12 Queen Square, London WC1N 3BG, UK

e-mail:a.mechelli@fil.ion.ucl.ac.uk

$\dagger$ Fondazione Santa Lucia, Roma 00179, Italy

1. Kim, K. H., Relkin, N. R., Lee, K.-M. \& Hirsch, J. Nature 388, 171-174 (1997)

2. Ashburner, J. \& Friston, K. J. NeuroImage 11, 805-821 (2000).

3. Good, C. D. et al. NeuroImage 17, 29-46 (2002).

4. Golestani, N., Paus, T. \& Zatorre, R. J. Neuron 35, 997-1010 (2002).

5. Poline, J. B. et al. NeuroImage 4, 34-54 (1996).

6. Warburton, E. A. et al. Brain 119, 159-179 (1996).

7. Maguire, E. A. et al. Proc. Natl Acad. Sci. USA 97, 4398-4403 (2000).

8. Draganski, B. et al. Nature 427, 311-312 (2004)

Supplementary information accompanies this communication on Nature's website,

Competing financial interests: declared none.

\section{brief communications arising online}

\section{www.nature.com/bca}

Mouse transcriptome: Neutral evolution of 'non-coding' complementary DNAs

J. Wang, J. Zhang, H. Zheng, J. Li, D. Liu, H. Li,

R. Samudrala, J. Yu, G. K.-S. Wong

(doi:10.1038/nature03016)

Reply: Y. Hyashizaki (doi:10.1038/nature03017) 